\section{Iron oxide nanoparticles coated with $\beta$-cyclodextrin polluted of Zea mays plantlets}

\author{
Mihaela Răcuciu \\ Lucian Blaga University, Faculty \\ of Science, Sibiu, Romania
}

\begin{abstract}
The present experimental investigation is focused on the study of assimilatory pigments and nucleic acid levels in young plants intended for agricultural use (Zea mays) in presence of water based magnetic fluid added in culture medium. The magnetic fluid was constituted by coating the nanosized magnetic nanoparticles (with $10.55 \mathrm{~nm}$ average value of the physical diameter) with $\beta$-cyclodextrin $\left(\mathrm{C}_{42} \mathrm{H}_{70} \mathrm{O}_{35}\right)$ and further dispersion in water. After germination, various volume fractions (between 10 $\mu \mathrm{L} / \mathrm{L}$ and $500 \mu \mathrm{L} / \mathrm{L}$ ) of the magnetic fluid was added daily in the culture medium of Zea mays plants still at their early ontogenetic stages. Toxicity symptoms leaded to brown spots covering the leaf surface for the highest magnetic fluid volume fractions used, a putative oxidative stress generated by iron excess treatment. Relatively small volume fraction of magnetic fluid solutions induced the increase of chlorophyll a level (up to 38\%), the main photosynthesis pigment, as well that the nucleic acid level (up to 57\%) in Zea mays plantlets. All volume fractions of magnetic fluid solutions analyzed may have severe disruptive effects such as the ratio chlorophyll a/chlorophyll $b$ (about $50 \%$ decreasing).
\end{abstract}

\section{Introduction}

The attention of the biophysics researcher is more and more headed for the magnetic nanoparticles functionalized with biocompatible surfactants, that prevents the magnetic nanoparticles aggregation and makes it suitable for biomedical applications. The magnetic fluids are two-phase systems, consisting of nanosized magnetic nanoparticles coated with a suitable surfactant and dispersed in a fluid. ${ }^{1}$ For biocompatible magnetic fluids, the core material is mostly magnetite or maghemite and the non-magnetic shells are made of biocompatible or even biodegradable compounds. A new generation of biocompatible magnetic fluids is composed of magnetic nanoparticles coated with cyclodextrins. ${ }^{2}$

The interest of the studying biological effects induced by the magnetic fluids in cultured microorganisms and vegetal organisms ${ }^{3,4}$ as well as animals ${ }^{5}$ has increased. The biological interest on the magnetic fluids effects in living organisms represents an important application field of magnetic nanoparticles, mainly for biotechnological use.

Special attention was paid to genetic effects of magnetic fluids such as induction of chromosomal aberrations in young vegetal plants. ${ }^{6,7}$ Regarding the influence of magnetic fluids on the photosynthesis process, a small number of studies is dedicated, ${ }^{4,8-10}$ which revealed a stimulatory effect on the photo-assimilatory pigments content leading to stimulatory effects on the plants growth. This stimulatory effect may be explained on the basis of iron importance in the vegetal organisms. ${ }^{11,12}$ The iron oxides from magnetic fluid composition (magnetite or maghemite nanoparticles) could be a source of colloidal iron for the plant development on a various volume fractions of the magnetic fluid supplemented culture medium. The biosynthesis of siderophore ${ }^{13}$ was assumed to be stimulated by the ferrous and ferric iron from magnetic fluid ferrophase. The present experimental investigation is focused on the study of assimilatory pigments and nucleic acid levels in young plants intended for agricultural use (Zea mays) in presence of water based magnetic fluid stabilized with $\beta$-cyclodextrin $\left(\mathrm{C}_{42} \mathrm{H}_{70} \mathrm{O}_{35}\right)$ in plants culture medium. The natural $\beta$-cyclodextrin $\left(\mathrm{C}_{42} \mathrm{H}_{70} \mathrm{O}_{35}\right)$ is natural product formed during bacterial digestion of cellulose and consists of seven glucopyranose units (Figure 1). The cyclodextrins can be topologically represented as toroids with the larger and the smaller openings of the toroid exposing to the solvent secondary and primary hydroxyl groups respectively, thus, the interior of the toroids is not hydrophobic, but considerably less hydrophilic than the aqueous environment and thus able to host other hydrophobic molecules. In contrast, the exterior of the toroids is sufficiently hydrophilic to impart cyclodextrins water solubility. The formation of the inclusion compounds greatly modifies the physical and chemical properties of the guest molecule, mostly in terms of water solubility. This is the argument why cyclodextrins have attracted much interest in many fields, especially pharmaceutical applications: because inclusion compounds of cyclodextrins with hydrophobic molecules are able to penetrate body tissues and these can be used to release biologically active compounds under specific conditions. ${ }^{14}$

\section{Experimental}

\section{Magnetic nanoparticles}

The water based magnetic fluid was constituted by coating the nanosized iron oxide
Correspondence: Mihaela Răcuciu, Lucian Blaga University, Faculty of Science, Sr.I.Ratiu Str.No.5 7, Sibiu, 550024, Romania.

E-mail:mracuciu@yahoo.com

Key words: assimilatory pigments, $\beta$-cyclodextrin, ferrofluid, iron oxide nanoparticles, magnetic fluid, nucleic acids young plants.

Received for publication: 1 September 2011. Revision received: 12 December 2012.

Accepted for publication: 22 February 2012.

This work is licensed under a Creative Commons Attribution NonCommercial 3.0 License (CC BYNC 3.0).

(C) Copyright M. Răcuciu, 2012

Licensee PAGEPress, Italy

Nanotechnology Development 2012; 2:e6

doi:10.4081/nd.2012.e6

nanoparticles with $\beta$-cyclodextrin $\left(\mathrm{C}_{42} \mathrm{H}_{70} \mathrm{O}_{35}\right)$ and further dispersion in water. ${ }^{15}$ Saturation magnetization of the magnetic fluid was of $2800 \mathrm{~A} / \mathrm{m}$, the ferrophase volume fraction was of $2.3 \%$ while the average particle diameter, obtain by transmission electron microscopy (TEM) analysis, was equal to $12.3 \mathrm{~nm}$. TEM microphotographs showed that the size of $\beta$ cyclodextrin coated iron oxide nanoparticles ranges between 1 and $30 \mathrm{~nm}$ (Figure 2), exhibiting mostly spherical shape. The ferrophase content was of $2.36 \cdot 10^{16}$ nanoparticles within the initial magnetic fluid sample.

\section{Biological material}

Seeds from a single plant (in order to diminish the putative genophond variations) were let to germinate on deionized watered porous paper support in Petri dishes (each sample was compound of 50 seeds) in darkness and suitable temperature. After germination, daily supply with $15 \mathrm{~mL}$ magnetic fluid aqueous suspension per dish of sample was carried out for 12 days, plant growth being conducted in controlled conditions of temperature $\left(22.00 .5^{\circ} \mathrm{C}\right)$, illumination (dark/light cycle: $14 \mathrm{~h} / 10 \mathrm{~h}$ ) and $90 \%$ humidity into a climate room. Water based magnetic fluid was added daily in various volume fractions (10 $50-100-150-200-250$ and $500 \mu \mathrm{L} / \mathrm{L})$ in the culture medium of Zea mays plants in their early ontogenetic stages.

\section{Analysis methods}

After 12 days of plant growth, the assimilatory pigments (chlorophyll a, chlorophyll b and total carotenoid pigments) and average nucleic acids (DNA and RNA) levels in the green tissues of all experimental samples as well as in the control plants were assayed by spectrophotometric methods using a JASCO V530 spec- 
trophotometer UV-VIS device provided with quartz cells of $1 \mathrm{~cm}$ width. The assay of the assimilatory pigments extracts (in $80 \%$ acetone) was performed following the Lichtenthaler and Welburn's method, ${ }^{16}$ while the assay of nucleic acid level (in perchloric acid 6\% extracts) was carried out accordingly to modified Spirin's method. ${ }^{17,18}$ Spectrophotometric measurements were performed at the wavelengths of: $663 \mathrm{~nm}, 646 \mathrm{~nm}$ and $470 \mathrm{~nm}$ (versus acetone $80 \%$ ) for the assay of chlorophylls (Chla, Chlb) and carotenoids pigments (Car) from green tissues and, at $260 \mathrm{~nm}$ and $280 \mathrm{~nm}$ (versus perchloric acid 6\%) in the case of nucleic acids. For the calculation of photosynthetic pigments, the formulae from Lichtenthaler and Welburn ${ }^{16}$ were applied while in the case of nucleic acids calibration curves (based on the spectral readings to the mentioned wavelengths) were used. Biological material consisted of green tissue obtained by mixing up the green tissue from the all young plantlets grown from each experimental group (sample). We used the same amount of fresh green tissue mass for each experimental sample used in the analysis conducted in this study. Plant individual length was measured with $0.1 \mathrm{~cm}$ precision. The average lengths and the standard deviations were calculated for each batch of test seeds. The confidence interval was calculated for every batch of plantlets using the Student test, for the confidence level $\mathrm{P}=90 \%$. The data provided by the chlorophylls ratio offered the main insight into the photosynthesis complex processes since they revealed the response of the LHC II system (Light Harvesting Complex II) to the external stimuli. ${ }^{19}$ Statistic analysis of the experimental data resulted from the three repetitions of the whole experiment was accomplished by means of ANOVA test - applied using MsExcell soft package - to evaluate reliability of modifications induced by magnetic nanoparticles added in the culture medium of plants in comparison to the control ones as well as among the samples corresponding to different volume fractions solution added in culture medium, considering the significance criterion of 0.05 (P value).

\section{Results and Discussion}

The iron excess treatment is believed to generate oxidative stress in leaf cells. ${ }^{20}$ Toxicity symptoms lead to brown spots covering the leaf surface (Figure 3 ). We have noticed that toxicity symptoms led to brown spots covering the leaf surface for the higher volume fractions of magnetic fluid aqueous solutions ( $250 \mu \mathrm{L} / \mathrm{L}$ and $500 \mu \mathrm{L} / \mathrm{L})$, added by us in the plants culture medium in this experiment. In this case, photosynthesis may be greatly affected leading to decrease of the process rate. ${ }^{20}$ Figure 4 presents the average plants length for each sample development in the aqueous magnetic fluid solution presence in culture medium.

We found that the enhanced volume fraction of aqueous magnetic fluid solution added in culture medium have an inhibitory effect on the growth of the plantlets. The results for higher volume fractions of magnetic fluid solution (>150 $\mu \mathrm{L} / \mathrm{L})$ are statistically significant, as resulted from the average comparison with the lengths of the control, using the Student $t$ test. The contents of photosynthesis pigments (a and b chlorophylls and total carotenoids) in the green tissue of young Zea mays plantlets

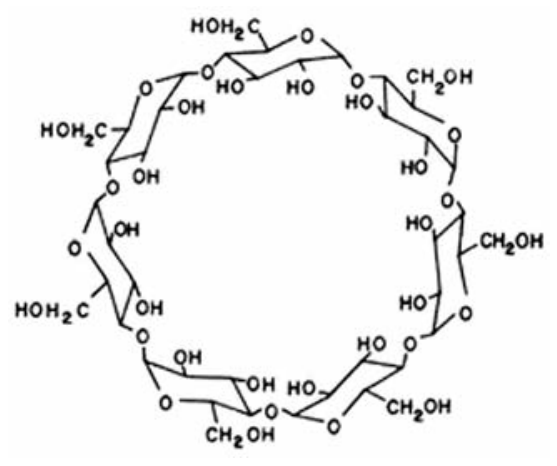

Figure 1. The chemical structure of $\beta$ cyclodextrin.

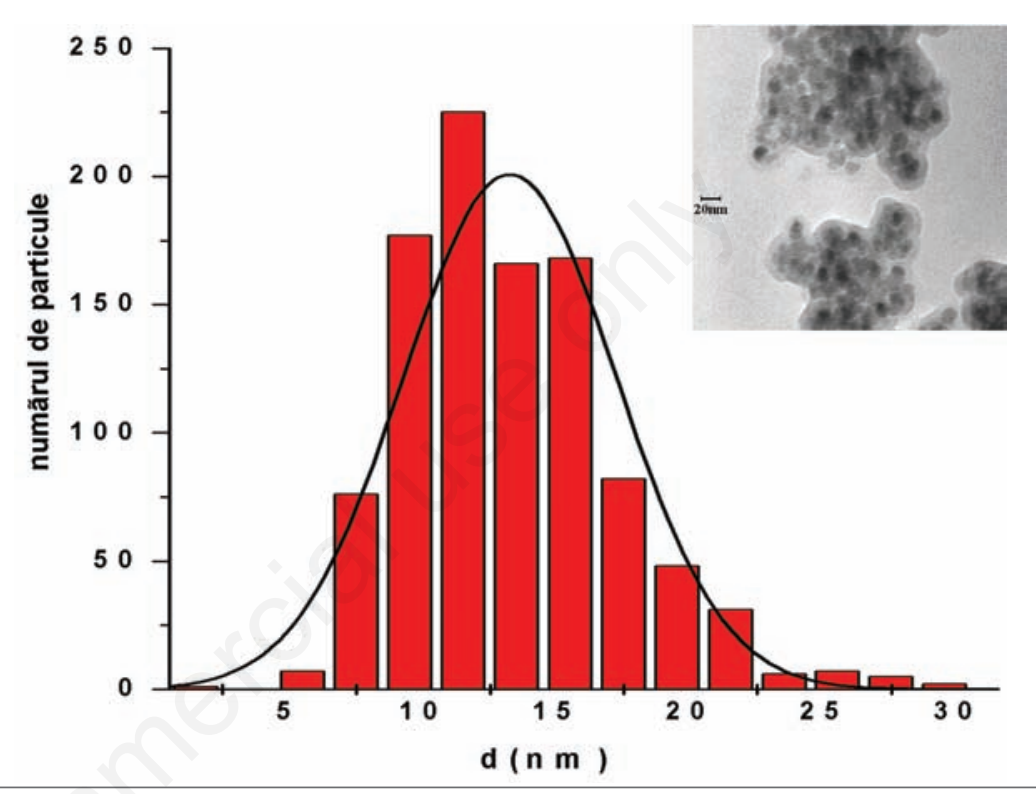

Figure 2. Histogram graph and transmission electron microphotograph of water based magnetic fluid sample.

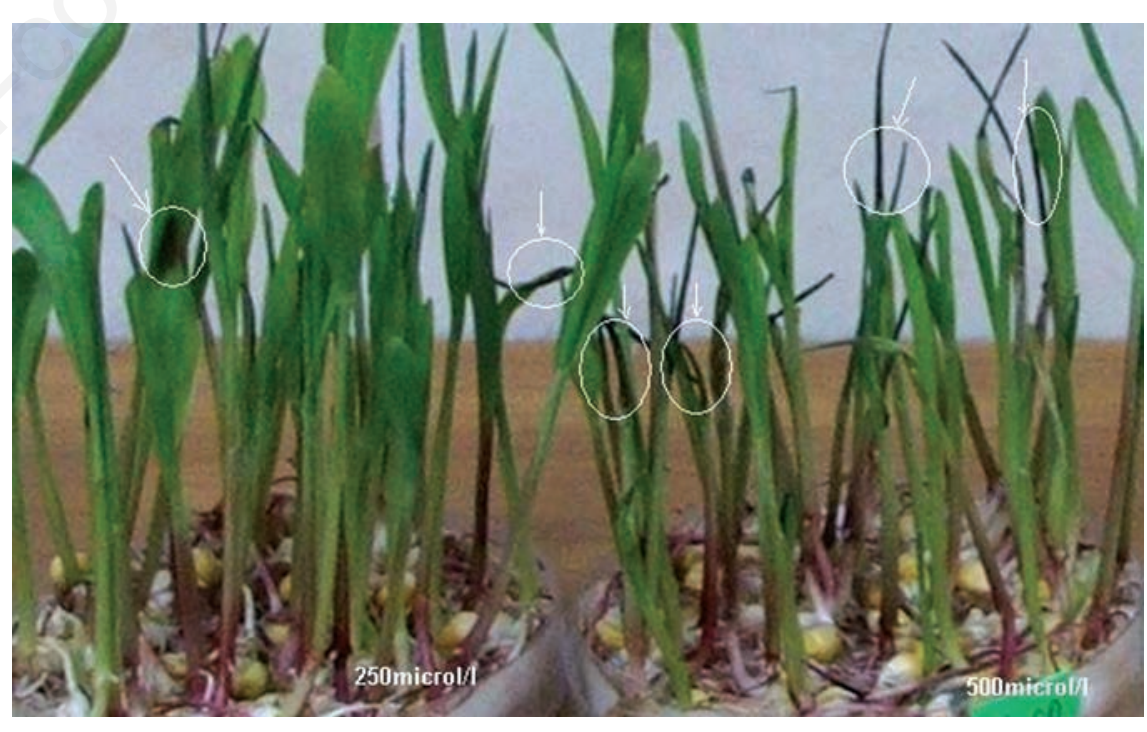

Figure 3. Photo of plants with possible toxic effects of magnetic fluids (samples with high volume fraction of magnetic fluid solution added in the culture medium). The toxicity symptoms were observed only after the ten days of growth to the samples with higher volume fractions of magnetic fluid aqueous solutions $(250 \mu \mathrm{L} / \mathrm{L}$ and $500 \mu \mathrm{L} / \mathrm{L})$ added by us in the plants culture medium. We were observed less damaged fractions of plant, possible due to of necrosis process. 
(aged of 12 days) for experimental samples in Figure 5 are presented.

The chlorophyll a level, the main photosynthesis pigment, was found increased for all volume fractions of magnetic aqueous solution (of $38.7 \%$ for the sample supplied with 250 $\mu \mathrm{L} / \mathrm{L})$ comparatively to the control sample (plants growth was performed only in deionised water presence) (statistically significant in relation to the threshold of 0.05 , an exception being the $500 \mu \mathrm{L} / \mathrm{L}$ supplied sample result). Similar response was get for the other two pigments analyzed (statistical significance was ensured relatively to the threshold of 0.05 , for all samples). The total assimilatory pigments contents have the same variation to the increase of volume fraction of magnetic fluid aqueous solution added in the culture medium of young plantlets that was observed for chlorophyll a level.

The best indicator upon the photosynthesis process efficiency is considered the chlorophylls ratio (chlorophyll a / chlorophyll b) ${ }^{19}$ which provides indirect information on the enzymatic aggregates of the Light Harvesting Complex II (LHC II) from the photosynthetic system II located in the chloroplasts membranes. The Zea mays response, in the magnetic fluid solutions presence (Figure 6), was decrease of chlorophylls ratio up to $50 \%$ with respect to the chlorophylls ratio when the volume fraction of the magnetic fluid solution increased from 10 to $500 \mu \mathrm{L} / \mathrm{L}$ (statistical significance was ensured relatively to the threshold of $\mathrm{P}<0.05$ ). This can be taken as a conclusive proof of the capacity of the $\beta$-cyclodextrin coated nanoparticles to influence the LHC II enzyme system.

The statistical analysis accomplished for the chlorophylls ratio (by applying the $t$-test to compare control (plants growth was performed only in deionised water presence) and test sample data) revealed statistic significance $(\mathrm{P}<0.01)$ for all samples under magnetic fluid influence. Linear correlation was evidenced between chlorophyll a (Chl a) and total carotenoid pigments (Car) levels, the correlation coefficient, $\mathrm{R}$, being 0.88 .

The average content of nucleic acids in young Zea mays plantlets after 12 days of grown under different volume fraction of magnetic fluid solution added in culture medium is presented in Figure 7. One can see that for increasing volume fraction of magnetic fluid solution the nucleic acid biosynthesis is inhibited (about 57\%) in comparison to the control sample. Applying the $t$-test to compare control (plants growth was performed only in deionised water presence) and test sample, data for the average nucleic acid level statistic significance $(\mathrm{P}<0.05)$ was found for all samples under magnetic fluid influence. Since a presumption of magnetic fluid supply interference with the nucleic acid biosynthesis is needed, one could
Zea mays, $\mathrm{P}=90 \%$

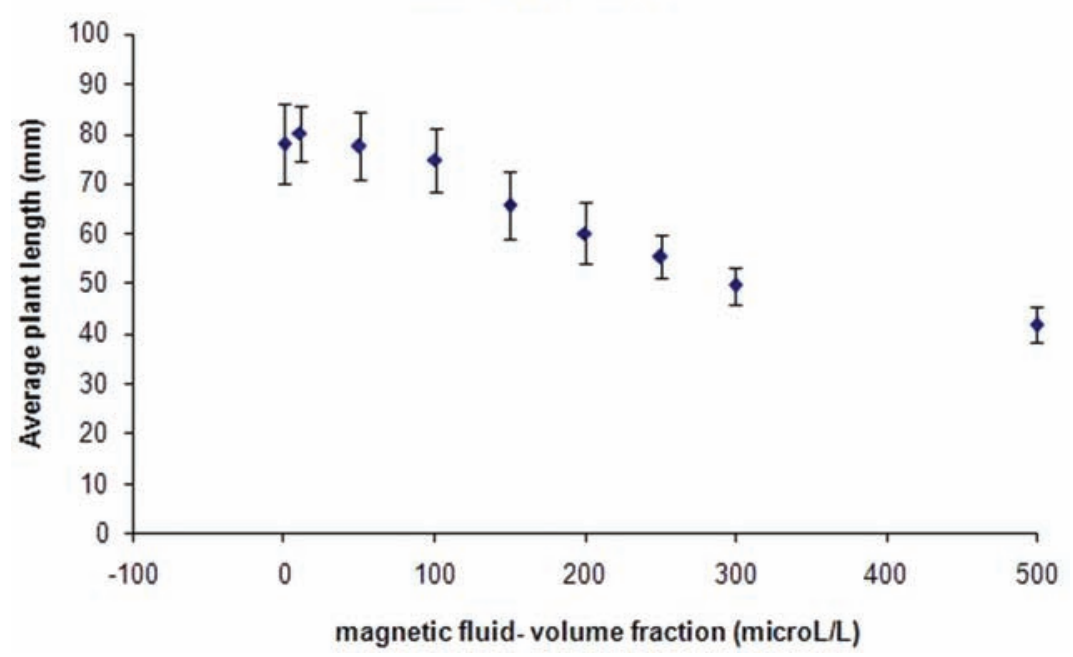

Figure 4. The average length versus volume fraction of magnetic fluid solution added in culture medium.

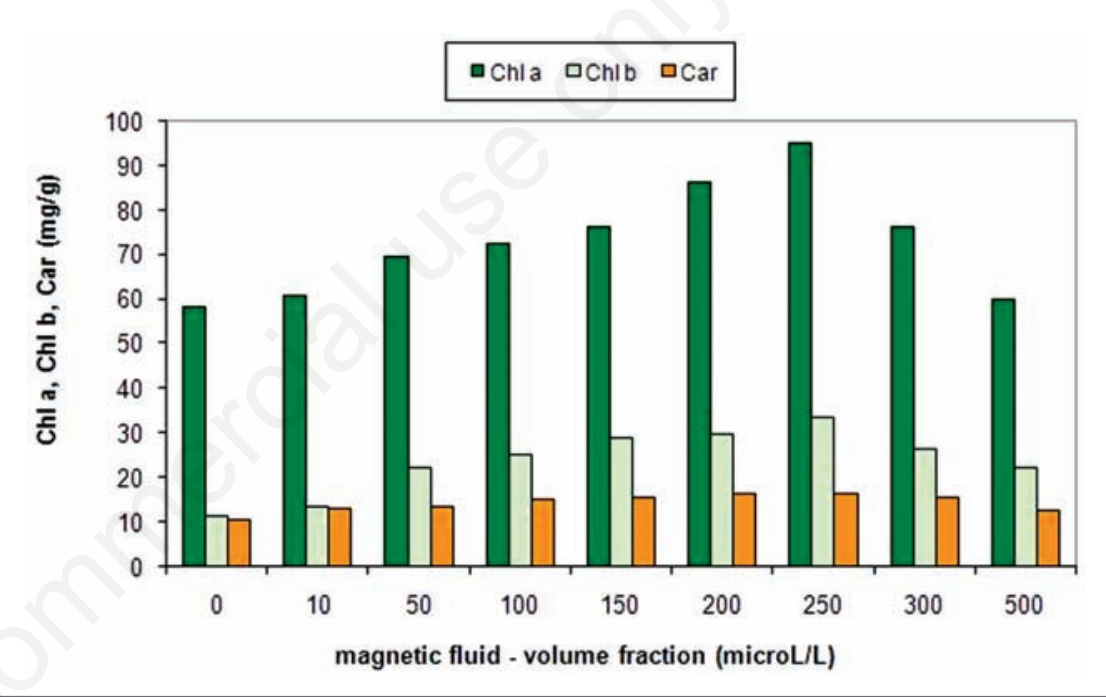

Figure 5. Assimilatory pigments level in Zea mays plantlets versus volume fraction of magnetic fluid aqueous solutions ( $\mathrm{Chl}$ a -the content of chlorophyll a, Chl b-the content of chlorophyll b, Car-the content of total carotenoid pigments).

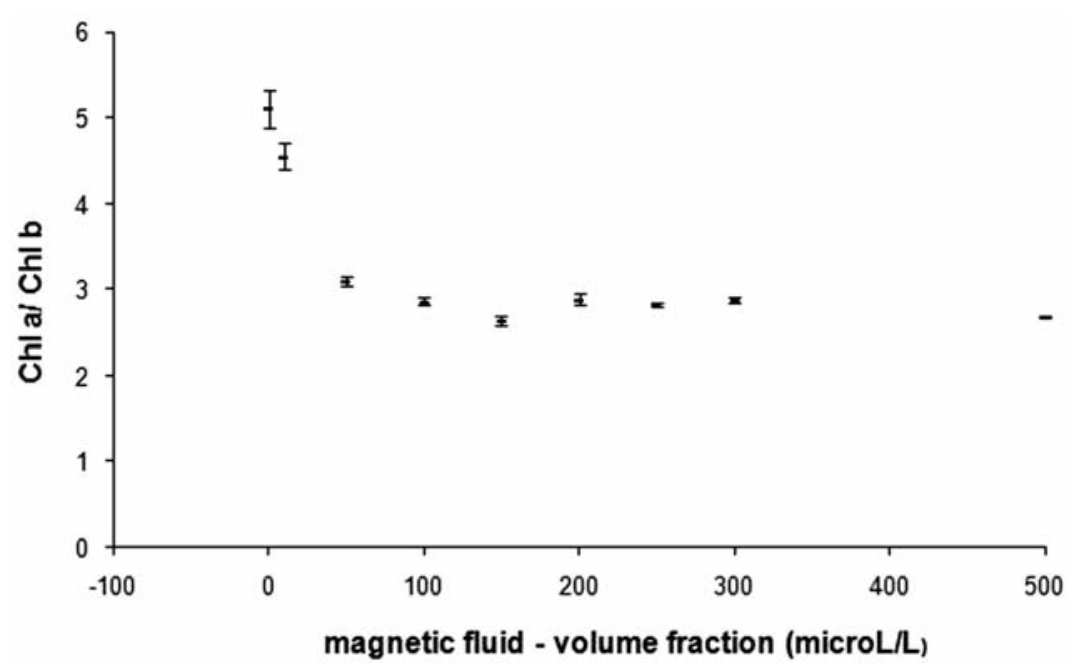

Figure 6. The effects of volume fraction of magnetic fluid solution added in the plants culture medium on chlorophylls ratio $(\mathrm{Chl} \mathrm{a} / \mathrm{Chl} \mathrm{b})$. 
imagine that the ferrophase could penetrate the nuclear membrane but the existence of extra-nuclear DNA and RNA need to be also taken into account. In this frame, the DNA from the chloroplasts is the most probable target of magnetic fluid effect in this experiment.

We presume that the iron oxides, provided by the magnetic nanoparticles from magnetic fluid ferrophase, could interfere with the complex redox reactions involved in the photosynthesis process. Furthermore, the iron uptake in the form of iron chelates, known as phytosiderophores, is another supposition that could be invoked when the magnetic fluid influence on the photosynthesis process is discussed since the putative siderophores presence in the tylakoidal membranes could result in some changes during the biochemical reactions from the vegetal cells. Other scientific studies have reported that iron treatment of Zea mays induced ferritin protein accumulation in roots and leaves over a period of 3 days. ${ }^{21}$

One may point out that through treatments of seeds, seedlings or mature plants with magnetic fluid solution, the magnetic nanoparticles penetrate into different plant structures where they could develop a distinct local magnetic field in a small volume with a ray of several nanometers. Plasmodesmata are small channels that directly connect the cytoplasm of neighboring plant cells to each other, establishing living bridges between cells, being important in cellular communication.22 Thus, ions, smaller molecules (e.g. monosaccharides) and water may move into, out of, within and between plant cells, pass through plasmodesmata by diffusion without the need for additional chemical energy. ${ }^{23}$ The diameter of plasmodesmata tube may varies between 20 and $200 \mathrm{~nm}$ and is on average of $50 \mathrm{~nm}$, but minor differences in their diameter being a result of the arrangement of proteins. ${ }^{24}$ The proteins are able to change their conformation to increase or decrease their size exclusion limit, thus the plasmodesmata may inhibit particular molecules to pass from one to another cell. In the case of cell-to-cell transport of viruses, plasmodesmata can be dilated with the help of movement proteins. ${ }^{25}$ Changes of the ultrastructure of the plasmodesmata tube have not been reported in infected plants. ${ }^{26}$

The magnetic nanoparticles (diameter size around $10 \mathrm{~nm}$ ) could have the ability of biomembrane penetration; or they could remain embedded in bio-membranes or in the cell cellulose wall, so that their superparamagnetic properties could influence local the transmembrane ion flows (magnetic influence on the ion channels). From the magnetic viewpoint, though the magnetic moments of the ferrophase nanoparticles are aligning parallel to the direction of an external magnetic field generating a significant total magnetic moment, however when the strength of the magnetic field is very small or even absent, aggregates will not provide a magnetic force.

\section{Conclusions}

Relatively small volume fraction of magnetic fluid solutions $(<500 \mu \mathrm{L} / \mathrm{L})$ added in the culture medium may induce the increase of chlorophyll a level, the main photosynthesis pigment, up to $38 \%$ as well that the nucleic acid level, up to 57\%, in Zea mays plantlets during their first days of life. All volume fraction of magnetic fluid solutions analyzed $(<500 \mu \mathrm{L} / \mathrm{L})$ have severe disruptive effects such as the ratio chlorophyll a/chlorophyll b (about 50\% decrease). We might say that $\beta$-cyclodextrin coated iron oxide nanoparticles water solution addition in the plant culture medium represented a source of iron and, possibly, magnetic energy that influenced in different ways the young Zea mays plantlets in the first days of life.

These experimental data are some preliminary results of my research in this field; in future will be started a complex experimental research to elucidate the influence mechanism problems, because the mechanism of magnetic nanoparticles on plant growth is unknown yet.

Different magnetic fluid type was induced different effects about plant development in early ontogenetic stages. The addition of water based magnetic fluid ( $\beta$-cyclodextrin coated iron oxide nanoparticles) solutions with different volume fraction presence in the culture medium of freshly germinated seeds was able to stimulate the plant proliferation in compar- ison to the control sample (seeds germination was able to in deionised water presence) and to induce an very low percentage for all analyzed samples $(<1.1 \%)$ of various types of chromosomal aberrations (e.g., micronucleus, inter-chromatin bridges, retard chromosome formation, chromosome fragmentation). ${ }^{27}$ Might presume that, some chromosomal aberrations induced by suitable volume fraction of biocompatible magnetic fluid solutions in plants culture medium could persist in the next generations, so that some phenotypic characters could be modified. These modifications could be observed following the plant growth, some of them being benefic for the cultivation of this agricultural species with major role in people life. Though presenting the capacity of inducing the cell nucleus response (widely assessed to genotoxicity) the effects of low level of nanosized magnetite in vegetal tissues should not compulsory result in damaging plant growth. As known, toxicity is related, on a side to the nature of chemical agent and the order of magnitude of its concentration in the living body while, on another side, it is dependent on the nature and sensitivity of the receptor biological system (low level chemical or physical factors being generally expected to have stimulatory influence).

The magnetic nanoparticles may be internalized in the vegetal tissue and may have not only a chemical but also a magnetic influence on the enzymatic structures implied in the different stages of the photosynthesis reactions. Finally, one should consider that possible biotechnological tool in the plant culture could be designed based on suitable magnetic fluid concentration range.

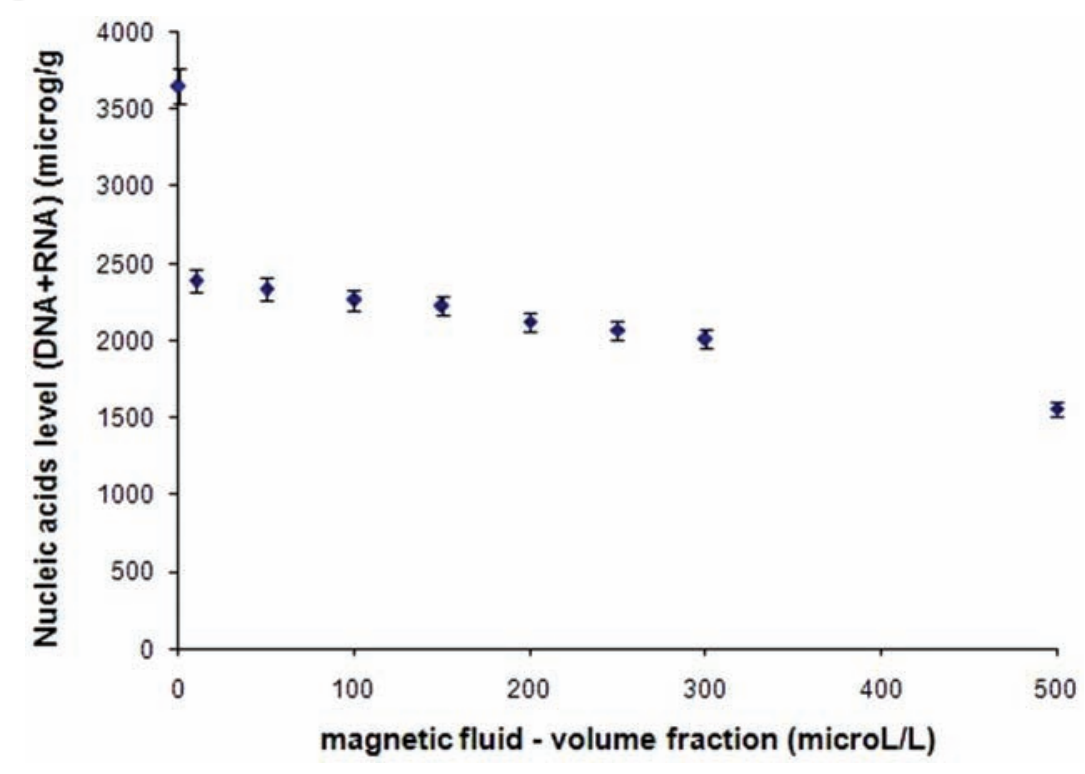

Figure 7. The average nucleic acid levels in Zea mays plantlets under magnetic fluid nanoparticles influence added in the plants culture medium. 


\section{References}

1. Rosensweig RE. Ferrohydrodynamics. Cambridge: Cambridge University Press, 1985.

2. Gansau C, Buske N, Götze T. Magnetic fluid (shell: carboxylated cyclodextrins) and process for preparation. DE patent pending 101540 16A1, WO/02-3035113A1.

3. Manoliu AL, Antohe L, Creanga DE, Cotae C. The influence of the petroleum ferrofluids upon the cellulosolytic fungi Chaetomium globosum Kunze: Fr. J Magn Magn Mater 1999;201:446-8.

4. Răcuciu M, Creangă DE. Influence of water based ferrofluid upon chlorophylls in cereals. J Magn Magn Mater 2007;311:291-4.

5. Goovaerts MJ, Dhaene J, Lacava ZGM, et al. Biological effects of magnetic fluids: toxicity studies. J Magn Magn Mater 1999;201: 431-4.

6. Pavel A, Trifan M, Băra II, et al. Accumulation dynamics and some cytogenetical tests at $\mathrm{C}$. majus and P. somniferum callus under the magnetic liquid effect. J Magn Magn Mater 1999;201:443-5.

7. Răcuciu M, Creangă D. Cytogenetic changes induced by aqueous ferrofluids in agricultural plants. J Magn Magn Mater 2007; 311:288-90.

8. Voica C, Polescu L, Lazar DA. The influence of the magnetic fluids on some physiological processes in Phaseolus Vulgaris. Rev Rom Biol 2003;48:9-15.

9. Pintilei M, Oprica L, Surleac M, et al. Enzyme activity in plants treated with mag- netic liquid. Rom J Phys 2006;51:221-6.

10. Răcuciu M, Creangă DE. TMA-OH coated magnetic nanoparticles internalized in vegetal tissue. Rom J Phys 2007;52:367-74.

11. Shenker M, Fan TW, Crowley DE. Phytosiderophores influence on cadmium mobilization and uptake by wheat and barley plants. J Environ Qual 2001;30:2091-8.

12. de Weger LA, van Arendonk JJ, Recourt K, et al. Siderophore-mediated uptake of $\mathrm{Fe} 3+$ by the plant growth-stimulating Pseudomonas putida strain WCS358 and by other rhizosphere microorganisms. J Bacteriol 1988;170:4693-8.

13. Adjimani JP, Emery T. Iron uptake in Mycelia sterilia EP-76. J Bacteriol 1987;169: 3664-8.

14. Becket G, Schep LJ, Tan MY. Improvement of the in vitro dissolution of praziquantel by complexation with alpha-, beta- and gamma-cyclodextrins. Int J Pharm 1999; 179:65-71.

15. Răcuciu M, Creangă DE, Bădescu V, Airinei A. Synthesis and physical characterization of magnetic nano-particles functionalized with $\beta$-cyclodextrin. J Optoelectron Adv Mater 2007;9:1530-3.

16. Lichtenthaler HK, Wellburn AR. Determinations of total carotenoids and chlorophylls $a$ and $b$ of leaf extracts in different solvents. Biochem Soc Transact 1983;11: 591-59.

17. Spirin A. [Spectrophotometric determination of total nucleic acids.] Biokhimiia 1958;23:656-62. [Article in Russian].

18. Struchkov VA, Strazhevskaya NB, Zhdanov RI. DNA-bound lipids of normal and tumor cells: retrospective and outlooks for func- tional genomics. Bioelectrochemistry 2002 ; 58:23-30.

19. Ort D, Whitmarsh J. Photosynthesis. Encyclopedia of Life Sciences. London: Macmillan; 2001.

20. Kampfenkel K, Van Montagu M, Inze D. Effects of iron excess on nicotiana plumbaginifolia plants (implications to oxidative stress). Plant Physiol 1995;107: 725-35.

21. Lobreaux S, Massenet 0 , Briat JF. Iron induces ferritin synthesis in maize plantlets. Plant Mol Biol 1992;19:563-75.

22. Jorgensen RA, Lucas WJ. Teaching resources. Movement of macromolecules in plant cells through plasmodesmata. Sci STKE 2006;21:323.

23. Epel BL. Plasmodesmata: composition, structure, and trafficking. Plant Mol Biol 1994;26:1343-56.

24. Rabaey D, Lens F, Smets E, Jansen S. The micromorphology of pit membranes in tracheary elements of ericales: new records of tori or pseudo-tori? Ann Bot 2006;98:943-51.

25. Oparka KJ. Getting the message across: how do plant cells exchange macromolecular complexes? Trends Plant Sci 2004;9:3341.

26. Rudzinska-Langwald A, Kaminska M. Cytopathological evidence for transport of phytoplasma in infected plants. Acta Soc Bot Pol 1999;68:261-6.

27. Răcuciu M, Creangă DE. Cytogenetical changes induced by $\beta$-cyclodextrin coated nanoparticles in plant seeds. Rom J Phys 2009;54:25-131. 